\title{
Guest-Editorial
}

\section{High performance computing for mission-enabling space applications}

\author{
Charles D. Norton \\ Jet Propulsion Laboratory, California Institute of Technology, USA
}

Advanced modeling, simulation, and analysis are essential to JPL mission success. Spanning all phases from mission conceptual design, spacecraft systems engineering, instrument development, navigation, science data product generation, and public outreach, computing has become ubiquitous and the demands for increased capability are growing rapidly.

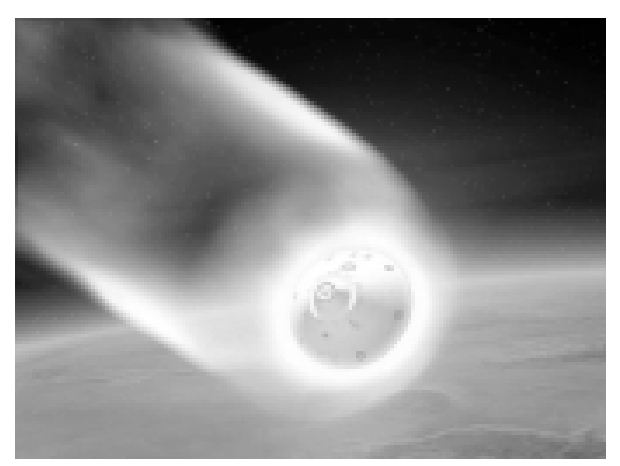

MER Entry-Descent-Landing (EDL) Monte-Carlo simulations used over 1,000,000 CPU hours for risk reduction during this critical sequence.

These demands are driven by factors tied directly to JPL's future mission set. For example, in some regimes physical testing cannot be performed and/or the cost of performing extensive validation in a physical testbed is prohibitively expensive. The design exploration space for project risk reduction, first principles simulation and data mining to discover new physical phenomena of interest, the need to produce high quality animations in a timely fashion, and design-trade studies for engineering analysis are also driving JPL's needs for High Capability Computing (HCC) to meet our mission challenges.

High Capability Computing involves the use of parallel computer systems (those with hundreds to thousands of processors) to solve a large problem more rapidly than a desktop computer system and/or to solve many variations of a specific problem simultaneously. This is an enabling capability for addressing the most advanced problems in science and engineering. High Capability Computing also includes development of computational technologies that both improve the fidelity of existing applications and spawn the generation of new ones. JPL maintains HCC systems within labs at the group, flight project, and institutional level. Furthermore, with various partners, JPL develops world-class science and engineering analysis software.

Many, particularly Earth observing missions, have used HCC in some form since JPL acquired initial systems in the early 1990s. Although one of the main drivers at that time was computational electromagnetics modeling even a few planetary missions, such as Magellan (1989), later benefited from HCC for comnbining radar surface topography data with digital elevation maps to produce visualizations of virtual flyovers of the Venetian surface. Such efforts span from re-processing of past data sets to very recent work such as the calculation of gravity fields, and other measurements, from the Gravity Recovery and Climate Experiment (GRACE) mission. As the technology and our infrastructure advanced so have our capabilities.

JPL and Caltech helped pioneer and establish the area of supercomputing in the mid-80s with development 


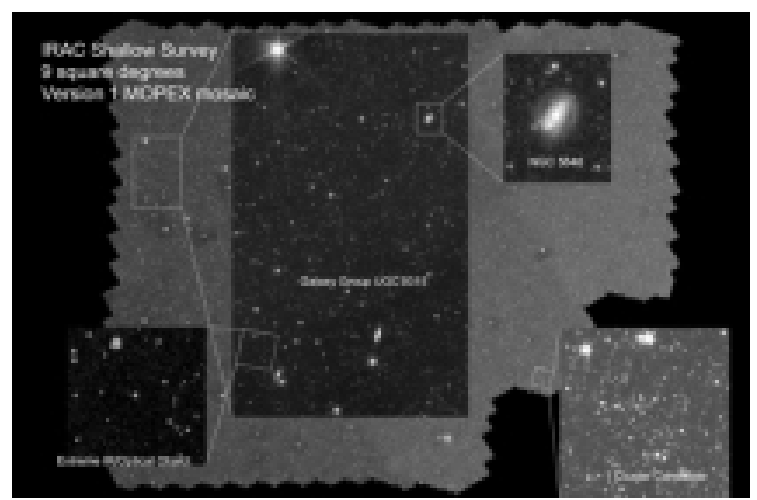

Efficient Mosaicing of Large-Area Spitzer Surverys, by Jacob et al.

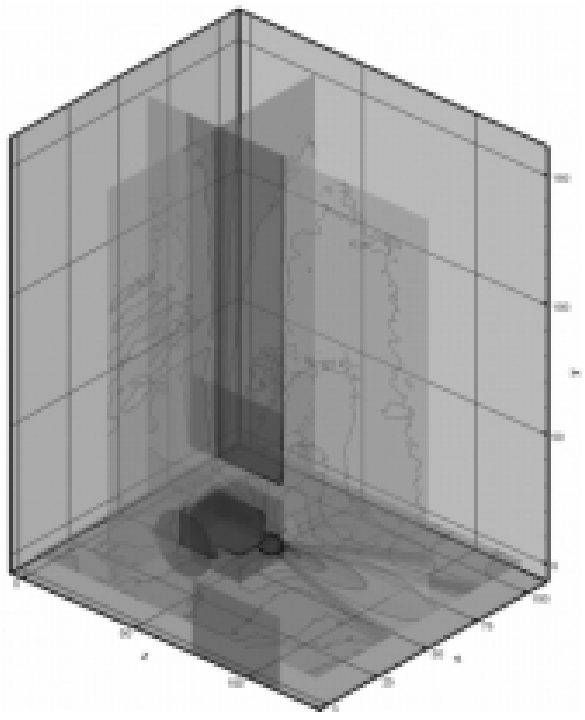

Electric Propulsion Plume Simulations by Wang et al.

of the Cosmic Cube (C. Seitz). This system used 64 nodes of $5 \mathrm{Mhz}$ Intel 8086 processors with $128 \mathrm{~K}$ bytes of storage using 700 watts of power for a manufacturing cost of $\$ 80,000$. The peak speed was 3 megaflops (MFlops). Over the years, as higher performance commercial systems and Beowulf cluster computers with gigaflops (GFlops) performance were produced, applications were developed for numerous projects and missions.

In 2005 JPL acquired a 1,024-processor cluster computer to both widen and deepen research and production computing capabilities for advanced modeling, simulation, and analysis in science and engineering. As the capability goals of JPL mission and technology projects grow in complexity, the utilization of high capability computing will allow more thorough and in-depth en-

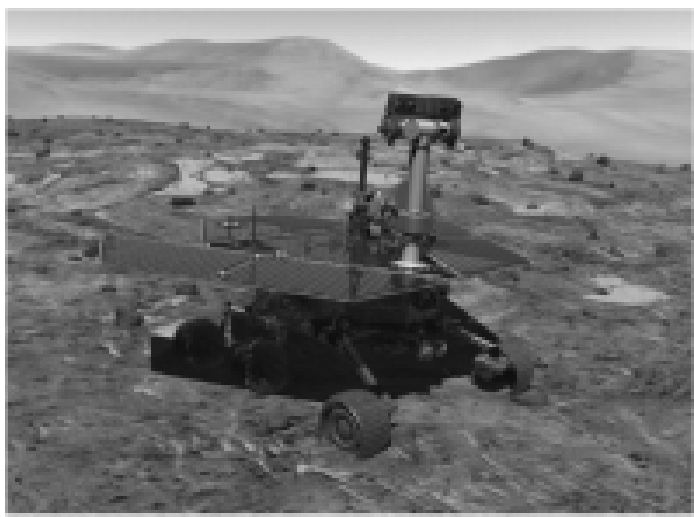

Large-Scale Rover Simulations by Madison et al.

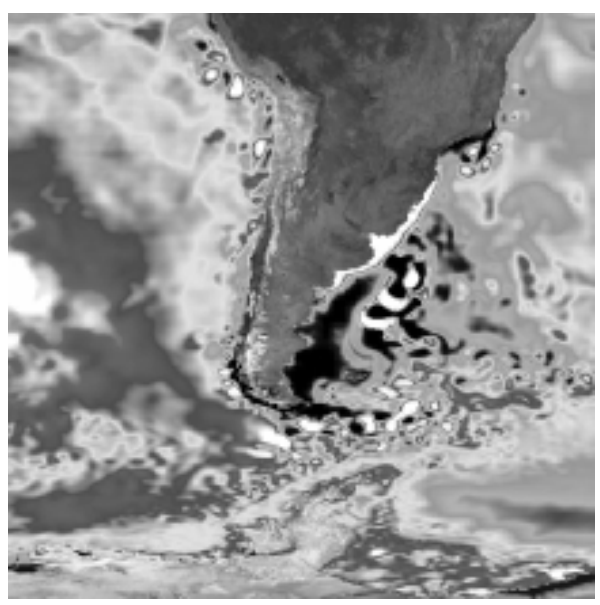

Global Ocean Simulation, by Hill et al.

gineering design trade-space analyses, faster and more accurate modeling for science understanding, virtual exploration of new mission and instrument concepts, and a more complete understanding of the interplay between the spacecraft and the space environment, to name a few. To achieve these goals JPL has placed a greater emphasis on fostering interdisciplinary interactions among discipline scientists, engineers, technologists, and project management, and those interactions have led to new capabilities that could only have been achieved with the use of high performance computing.

In this special issue we introduce a few of the new capabilities developed at JPL related to electric propulsion plume simulations, large-scale rover navigation simulations, large-scale mosaic generation from Spitzer surveys, and ocean/sea-ice interaction sensitivity studies. Numerous other applications have also been pursued to address our needs in computational science and engineering. 
Efficient Mosaicing of Large-Area Spitzer Surveys: This work demonstrates how HCC implementation of the MOPEX imaging mosaic software enables Spitzer scientists to optimize cosmic ray detection and produce improved Infrared Array Camera (IRAC) shallow survey mosaics to facilitate searches for brown dwarfs and other rare objects. The original mosaics required approximately 70 hours on a desktop system while the HCC version needs approximately 2 hours using a small portion of the Dell Cluster. Disk system speed is the performance-limiting factor for this data intensive application.

Electric Propulsion Plume Simulations: This work demonstrates how HCC enables complex engineering simulations in regimes where flight test data is limited. This capability is a first-principle based, and experimentally validated simulation for high-fidelity predictions of electric propulsion effects on spacecraft and science measurements. While there have been significant efforts in research on electric propulsion plume effects, investigating and predicting plume-spacecraft interactions in a vacuum tank remains a challenging endeavor due to the intrinsic complex nature of the physics, the difficulty of matching in-space conditions in a laboratory, and effects from test chamber and diagnostic techniques. On the other hand, the cost associated with performing space experiments and a lack of flight opportunity precludes using in-flight investigation to study most interaction scenarios. This capability to accurately predict such effects will be critical for all future missions using electric propulsion. The new software has enabled simulation of ion thruster plume interactions for the DAWN spacecraft configuration (including solar panels) and the NEXIS thruster. It represents the only in-flight data validated model to be published where $\mathrm{HCC}$ allows simulation within $10 \mathrm{~s}$ of hours rather than 1000s of hours.

Large-Scale Rover Simulations: This work demonstrates how HCC enables a new capability to run high- fidelity rover simulations. It allows the systematic exploration of system performance over a large parameter space under precisely controlled conditions. HCC simulation also allows testing under conditions not available in hardware testbeds because of expense, risk to the testbed, or simply physical impossibility. For rover surface terrain missions, such as MSL, HCC exploration of the large parameter space governing both the terrain environment and the rover, is essential for carrying out performance and risk studies for the autonomy capabilities onboard the rover and thousands of such tests can be conducted simultaneously. Thousands of mobility test cases can now be examined simultaneously using the Dell cluster as opposed to running a much smaller number of cases one at a time. In 2 hours, tests were performed that would require 5 days of continuous hardware testbed time, assuming no problems occurred on the testbed.

Global Ocean Simulations: This work contributes to a project called Estimating the Circulation and Climate of the Ocean, Phase II (ECCO2): High-Resolution Global-Ocean and Sea-Ice Data Synthesis. ECCO2 aims to produce increasingly accurate syntheses of all available global-scale ocean and sea-ice data at resolutions that start to resolve ocean eddies and narrow current systems, which transport heat, carbon, and other properties within the ocean. ECCO2 data syntheses are obtained by least squares fit of a global full-depth-ocean and sea-ice configuration of the Massachusetts Institute of Technology general circulation model (MITgcm) to the available satellite and in-situ data. The paper herein focuses on the computational aspects of reaching the technical objective of efficiently performing these global eddy-resolving ocean simulations.

High capability computing is essential to achieving many of JPL and NASA's future ambitious goals in space exploration as modeling and simulation is increasingly used to help drive testing, validate mission concepts, and improve science return. 

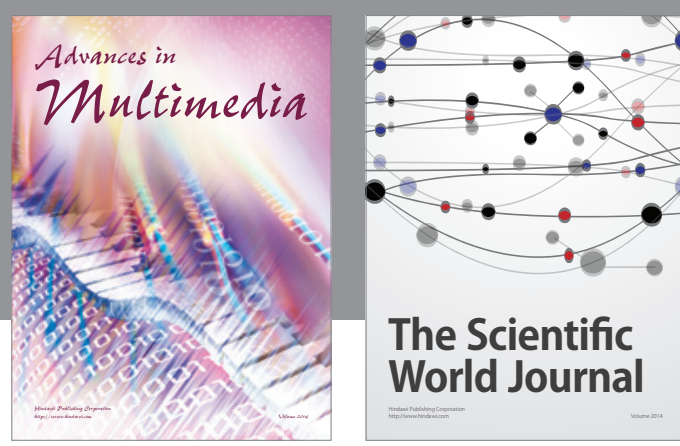

The Scientific World Journal
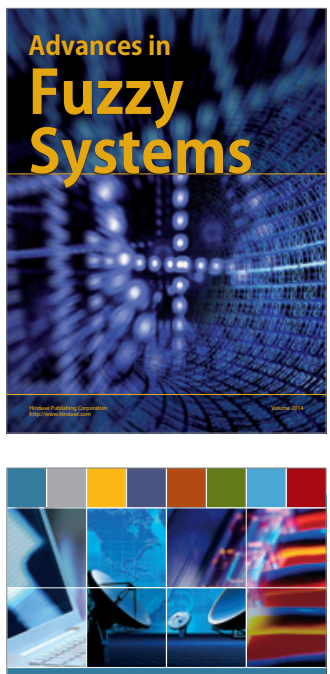

Computer Networks and Communications
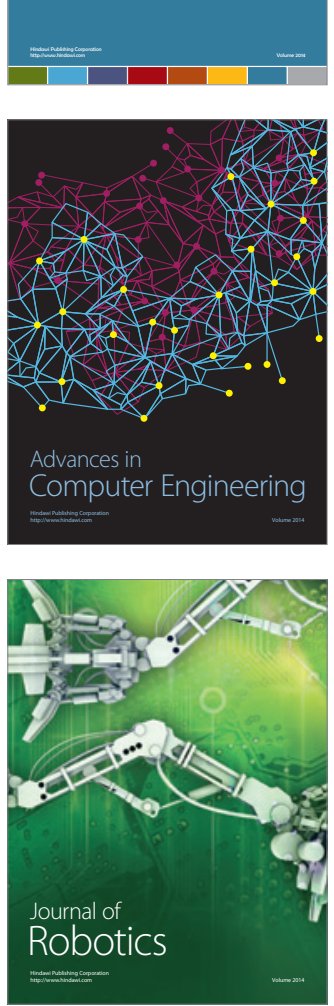
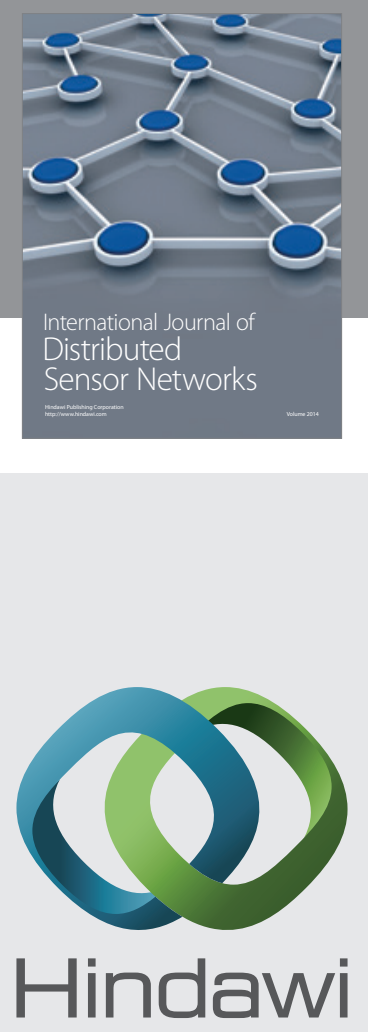

Submit your manuscripts at

http://www.hindawi.com
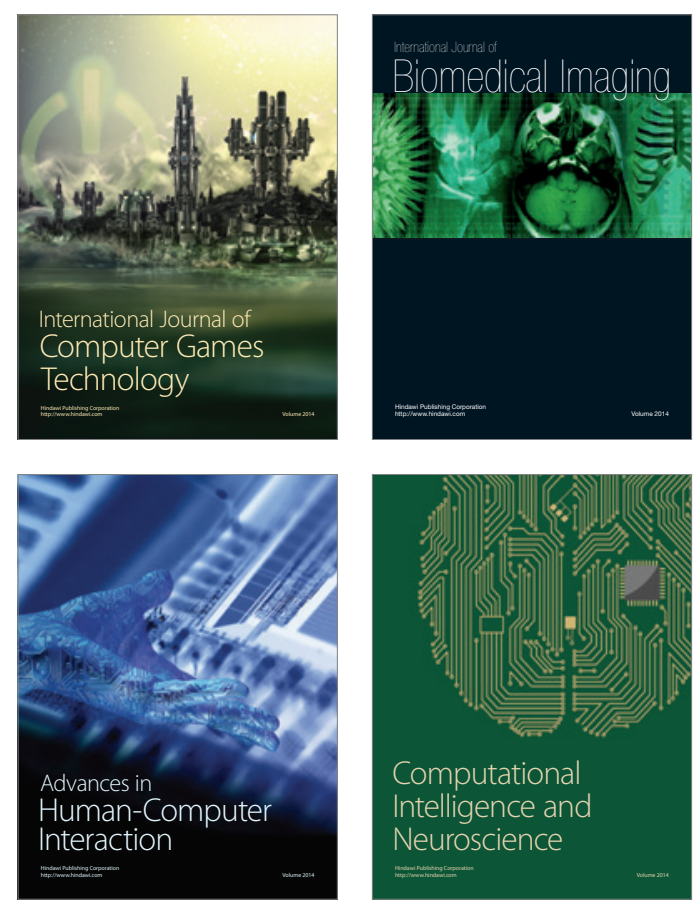
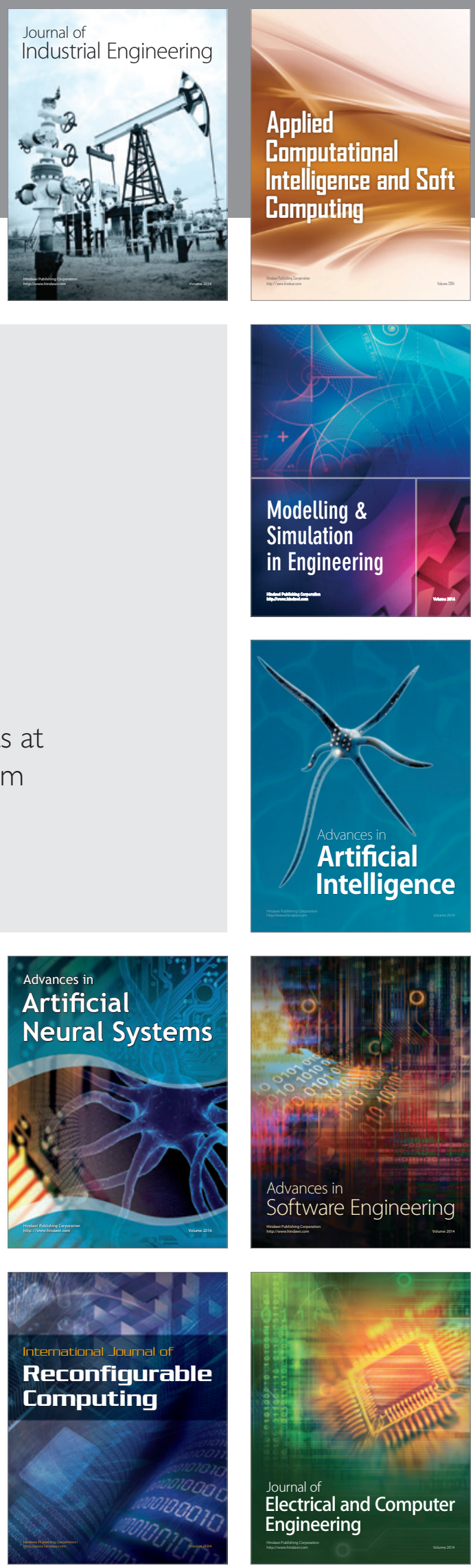